\title{
Questions for Classroom Response Systems and Teaching Instrumental Element Analysis
}

\author{
Gunnar Schwarz*
}

\begin{abstract}
Asking students questions is a central, although understudied and underappreciated, ingredient of teaching. Formative questioning provides many opportunities for teachers and students, e.g. to practice skills and receive feedback. Among other approaches, classroom response systems (CRSs), which run on the mobile electronic devices of students, facilitate such active engagement of students in the lecture hall. This paper presents an overview on questions for teaching with a focus on questions for CRSs and provides considerations and brief guidelines for the development of multiple-choice questions. Examples from a mid-sized analytical chemistry lecture illustrate additional challenges and different probes for potential misconceptions. Moreover, limitations of valid interpretation of students' responses are emphasized. This leads to a discussion of the value of incorporating prompts for justifications into questions.
\end{abstract}

Keywords: Analytical chemistry · Classroom response system · Misconceptions · Questions · Second-year undergraduate

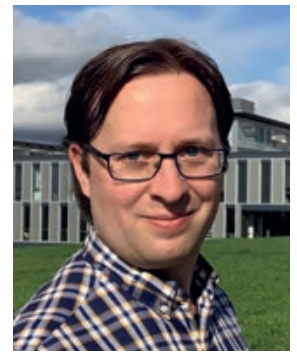

Gunnar Schwarz was born in 1983 in Berlin, Germany. He undertook his doctoral studies in the group of Michael Linscheid at Humboldt-Universität zu Berlin, where he advanced metal labelling tools that utilize DOTA chelate complexes for protein quantification. In 2014, Gunnar joined the group of Detlef Günther at ETH Zürich as a postdoc and worked on LA-ICP-TOFMS imaging and is dedicated to teaching analytical chemistry. Among the latter was the project seminar 'Methods in Quantitative Element Analysis' in 2018. He liked to ride his bicycles in and around Berlin. Yet, in Switzerland he rediscovered his passion for hiking.

\section{Introduction}

Questions, ${ }^{[1]}$ especially those in exams, drive the focus of learning for many students. It can be reasonably argued that it is more important whether and what a lecturer asks during a course than through which medium. By questioning, lecturers are able to emphasize, recap, or amend specific facts, concepts, relationships, processes, conflicts, controversies. Moreover, the feedback to question responses is vital in education; ${ }^{[2,3]}$ it can guide and support further instruction and learning with no or minor instant influence on grading (formative assessment), which is in contrast to feedback provided by exams (summative assessment). [4] Ideally, feedback acts in both directions, from lecturers to students and vice versa to adjust learning activities.

There are many articles on the utilization of classroom responses systems (CRSs) for chemical education. ${ }^{[5-9]}$ I characterized CRSs as interfaces between lecturers and students for question-answer sessions and proposed a framework for their implementation. ${ }^{[10]}$ Indeed, CRSs offer a unique set of features: While a show-of-hands and coded cards are able to actively engage a large number of students, depending on the system employed, CRSs are also able to handle responses anonymously and al- low short written responses to be collected from all students swiftly.

One may ask questions without a CRS, but cannot use a CRS without questions. Consequently, this paper will discuss challenges associated with questions and present examples. Although the focus is on multiple-choice questions (MCQs) for formative use with CRSs, similar issues arise when questions are put to students verbally, in writing, or out of class via learning management platforms like Moodle and remote learning. Hence, the fundamentals (see Supporting Information), examples, and insights offered here are more general in nature. Moreover, a recommendation for requesting justifications for option selection and conclusions about the 'meaningfulness' of questions with respect to the responses will be derived.

\section{Any more questions?}

There is a growing promotion of and interest in 'active learning'. Since all active learning approaches depend on questions it is somewhat astonishing that studies and educational literature on questions for educational purposes and question development for routine teaching are scarce.[11] MacArthur ${ }^{[12]}$ already criticized the general lack of information on clicker questions, i.e. CRS questions, in peer-reviewed articles and meetings. It comes as no surprise that the development and phrasing of questions was identified as a major hurdle for CRS implementation. ${ }^{[13]}$ Available MCQs (in data bases, ${ }^{[14]}$ textbooks, online sources, etc.) for analytical chemistry include mostly basics such as stoichiometry, $\mathrm{pH}$-related topics, titrations, etc. More advanced topics like instrumental or structural analysis are rarely covered. Moreover, questions found in textbooks are also not easily converted to MCQs.

While a general framework for questions in educational contexts is still absent, it seems expedient to provide a brief overview on central considerations. The Supporting Information expounds on the structure of questions, their cognitive dimension, best vs. correct reply options, and specifics to questions in CRSs. 
In brief, among other classifications, questions are commonly distinguished into closed- and open-ended questions (OEQs), depending on the answers deemed as suitable. In the context of CRSs, MCQs are most common and come in different formats (Table S2, Supporting Information). There is a strong endorsement for 'challenging problems' or so-called concept(ual) questions in the context of CRSs in the literature. ${ }^{[15,16]}$ The intention can be best understood by contrasting to what these questions ought not to be: promoting plain rote learning, recognition, or numerical problem solving. However, as outlined in the Supporting Information, whether a question can meet this standard is highly situation-related.

\section{The Challenge of Multiple-choice Question Development}

There is no broad agreement about the characteristics of meaningful questions for educational purposes in the literature. Beatty et al. ${ }^{[16]}$ noted that questions can be very useful for certain teaching approaches (e.g. Mazur's peer instruction $\left.{ }^{[15]}\right)$, but the same might fail for others. A challenging question might be helpful to introduce a topic, begin a well-put exercise, jump-start discussion, or as a useful diagnostic tool for formative assessment to stir the direction of learning. However, the same question could fail in other circumstances by introducing confusion that sabotages learning gains already achieved. In fact, MacKeachie ${ }^{[17]}$ notes "...the greater your experience in their construction, the longer it takes per [multiple-choice] item to construct a reasonably, fair, accurate, and inclusive question." (cited from ref. ${ }^{[18]}$ ) What constitutes a 'good question' depends on many factors.

Tactics for MCQ design was presented by Beatty et al. ${ }^{[16]}$ They advise to consider the pedagogic objective in terms of the content and process goals of a question. This provides a wellfounded starting point. The content goal focusses on the specific subject matter and the process goal on the skills the students are expected to employ to answer the question (compare to cognitive domain, Supporting Information). Both should generally be aligned with the overall course objectives or amended by other activities. Beatty et al. also mention the metacognitive goal, but it remained largely unclear how this may shape questions further, if the questions are not specifically aimed at such processes (e.g. "What did you learn today?" or inquiries for so-called muddiest points $\left.{ }^{[19]}\right)$. However, as Pappa et al..$^{[20]}$ point out metacognitive questions can be valuable incentives for students to develop skills by abstracting their approach to solve problems. Illustrative question examples can be found in the appendix of Pappa et al. ${ }^{[20]}$

The quality of distractors (incorrect options) in MCQs ensures that the presented problem goes further than recognition. The most often encountered flaw in MCQs is the implausible distractor. ${ }^{[21]}$ It has been shown that specific phrasing of questions and options is an important aspect. ${ }^{[22-24]}$ Questions seemingly unambiguous to experts were understood differently, but still in a reasonable manner, by novices. ${ }^{[22]}$ On the other hand, it is an (often implicit) objective of many courses that students are able to handle the terminology, models, and underlying assumptions. If this poses consistent difficulties, they are in turn candidates for meaningful, separate questions.

Distractors for multiple-choice tests in educational studies are often generated from interviews with students and MCQs are tested thereafter at a smaller scale before being employed. While this does not guarantee valid questions, ${ }^{[22]}$ this strategy is by far too expansive for routine users of CRSs. Answers given by students to OEQs in earlier exams and written exercises can serve as basis for distractors. After the key (best or correct option) has been phrased, the key can be varied for the distractors by exchanging specific terms, insertion of imperfect or insufficient information $^{[16]}$ or hypernyms ${ }^{[25]}$ and hyponyms. ${ }^{[26]}$

\subsection{General Recommendations for Multiple-choice Questions}

Most of the literature on MCQs focusses on diagnostic and summative assessments, which does not consider the formative use of CRSs. Four additional articles are especially noteworthy to be considered for MCQ development: Haladyna et al. ${ }^{[27]}$ provided guidelines in the form of 31 short demands for writing MCQs for classroom assessment. Towns ${ }^{[28]}$ offered guidelines for multiplechoice assessments with examples from general chemistry and information on post-exam item analysis to improve subsequent MCQ development. Clegg and Cashin ${ }^{[18]}$ presented an overview with recommendations on MCQ tests. Besides, Gierl et al. ${ }^{[29]}$ reviewed the literature and made a strong case for distractors as the 'Achilles' heel' of MCQs. Though developing MCQs is ever so often a creative process which does not follow a straight path, for the use with CRSs (i.e. not necessarily for exams) the following pooled recommendations to design MCQs seem most vital:

1. Start by selecting content and process goals. ${ }^{[16]}$

2. Write the stem first, then the key(s), then distractors; be certain that the best option is really the best.

3. The stem alone should be answerable without the options.

4. State the problem within the stem concisely, but completely.

5. Try to use typical errors, misunderstandings or false assumptions and interpretations of students as distractors.

6. Consider the time to read and process the question as well as formulating a response and reasoning.

7. Avoid (phrasing) repetition in the options, consider rephrasing of the stem instead.

8. Avoid negatives ('not', 'no'), if used, emphasize them with boldface, underlines, and/or capital letters.

9. Avoid making the key(s) longer than the distractors.

10. Avoid implausible distractors, but use familiar words (terminology) for distractors.

11. Avoid clues with terms like 'all', 'never', 'always', 'maybe', 'must', 'usually', etc.

12. Avoid grammatical inconsistency between options.

13. Avoid options like 'none of the above', 'all of the above', 'A and C', etc.

14. Check all again, recheck again, and evaluate after using the question in class.

Questions may include the additional option of abstention ("I don't know."). This provides a more realistic distribution of students' ability and counteracts guessing, but also eases the pressure of commitment and students opt for abstention at different levels of certainty. There are no universal recommendations for whether to use this option or not. However, in light of the formative aims of CRSs it is reasonable to include this option as this can reveal valuable information for the lecturer to conduct the class. If a large portion of a class indicates that they "don't know" the lecturer may ask for explanations, e.g. lack of background knowledge, from students. It is worth noting, that the difficulty of a question (e.g. the quotient of the average allocated points and the maximum points) is a post-hoc parameter and can only be estimated before the question is put.

\section{Discussion of Question Examples}

In the following, several examples for CRS questions are presented and discussed. In particular, the selection of questions was not only driven by usefulness, but to point out shortcomings of questions and interpretation of responses. All examples were taken from the quantitative element analysis part of the courses Analytical Chemistry I and II between fall 2017 and fall 2019. Topics of these courses include the analytical process, sampling and sample preparation, quantitative analysis and calibration, inductively coupled plasma optical emission spectroscopy (ICP-OES), and atomic absorption spectroscopy (AAS), X-ray fluorescence spectroscopy (XRF) and inductively coupled plasma 
mass spectrometry (ICP-MS). A more detailed description of the courses and the CRS approach is provided in ref. [10] and the CRS used in ref. [30]. All questions were translated from German, are numbered consecutively and formatted italic. The correct or best options are labeled with an asterisk $(*)$. The total number of votes $(N)$ and vote distributions are provided.

\subsection{From Open-ended Questions to Multiple-choice Questions}

The demanding search for suitable distractors can be circumvented or at least simplified if responses to OEQs (former exams, worksheets, etc.) from students are available. Some CRSs allow the creation of MC options from OEQ responses. After a first introduction to ICP-MS, students were shown an ICP mass spectrum of an arctic ice water sample (Fig. 1). Following some direct question-answer cycles around the signal at $\mathrm{m} / \mathrm{z}, 80$ and relative signal intensities, identifying it as the argon dimer $\left({ }^{40} \mathrm{Ar}_{2}^{+}\right)$, students were asked an OEQ via the CRS ("What causes the signal at $\mathrm{m} / \mathrm{z}$, 54?"). Students provided free text input as responses (data not recorded). The options for a second vote as a MCQ were selected according to their apparent frequency and included also the single somewhat correct response ' $\mathrm{N}+\mathrm{Ar}$ ' from a student. In the following years the options were written with the common nomenclature (e.g. ' $\mathrm{O}_{3}{ }^{+}$' instead of the student reply ' 3 times O'). Hence, the following options were used:

1. What causes the signal at $\mathrm{m} / \mathrm{z}, 54$ ? $(\mathrm{N}=59)$
$\begin{array}{lll}\mathrm{A}^{*} & \mathrm{ArN}^{+} & 7 \% \\ \mathrm{~B} & \mathrm{O}_{3}^{+} & 2 \% \\ \mathrm{C} & \left(\mathrm{H}_{2} \mathrm{O}\right)_{3}^{+} & 0 \% \\ \mathrm{D} & \mathrm{Cr}^{+} & 42 \% \\ E & \mathrm{Fe}^{+} & 42 \%\end{array}$

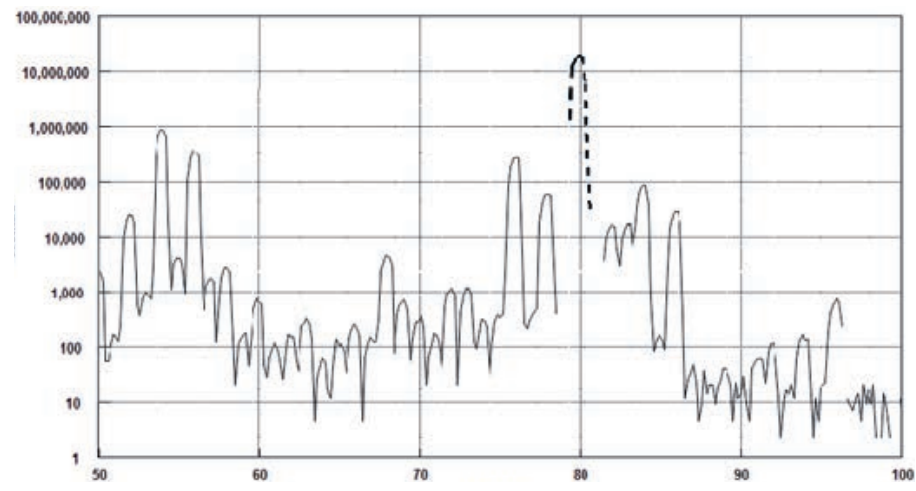

Fig. 1. ICP mass spectrum of an arctic ice water sample.

One year the lecturer inadvertently ruled out options D and $\mathrm{E}$ as viable options, which shifted the votes not towards option $\mathrm{A}$, but B and C (data not shown). The result served as a lead-in to discuss other plasma-background ion species. This mode was very useful to generate attractive distractors, but with the CRS ${ }^{[30]}$ used all options not intended for the MCQ have to be eliminated manually, which would take some time for a large audience and numerous responses.

\subsection{Identification of Potential Misconceptions and Peer Instruction}

Effective teaching takes misconceptions held prior or developed by students during the instruction into account and addresses them with appropriate teaching activities. ${ }^{[31]}$ However, in order to counteract misconceptions effectively, mere diagnosis via incorrect selection MCQ options is insufficient; students' reasoning has to be taken into consideration. CRSs provide an opportunity to assess both. An exit ticket (CRS questions put to the students at the end of a lecture) contained the following set of questions:

2. Assess the following statement. "The sensitivity of atom absorption spectrometry $(A A S)$ can be increased by increasing the irradiance of the light source." $(N=45)$

$\begin{array}{lll}\text { A } & \text { True. } & 36 \% \\ B^{*} & \text { False. } & 64 \%\end{array}$

3. Please justify your answer. (OEQ)

While most students correctly selected B, only 20 of those 29 provided at least one word as justification. Nine of those (45\%) were referring to the Beer-Lambert law or relative irradiance, thereby providing sound and subject specific reasoning. Common other justifications referred to different parameters, claiming influences on sensitivity. This question was revisited during the following lecture with similar questions and subsequent verbal explanations.

Responses provided by students to worksheet questions suggested that they consider an avoidable error as a systematic error. Although it might be argued that most systematic errors are somewhat avoidable, this is not congruent with the concept of systematic errors, as just a fleeting look at a burette to read the volume scale is an avoidable error but likely leads to an additional random error. After revision, a phrasing in the lecture slides was found which might have misled students to the assumption that the possibility for avoidance of an error is a criterion for systematic errors. During the following lecture (before the students had access to the sample solution to the worksheet) the same question and justifications were put to the students by the CRS without any prior explanation in order to test this potential misconception.

4. For digesting a sample to determine silicon you used hydrochloric acid which was contaminated with silicon. This led to ... $(N=53)$

$\begin{array}{lll}A & \text { a random error } & 6 \% \\ B^{*} & \text { a systematic error } & 92 \% \\ C & \text { no difference } & 2 \%\end{array}$

5. Why have you chosen this answer?

$(N=44$, only counting votes from B in question 4$)$

A This is an avoidable error. $\quad 16 \%$

B This affects all samples in the same manner. $84 \%$

C The effect is undirected. $0 \%$

$D \quad$ This depends on the executing person. $0 \%$

It should be noted that in case of question 5 the 'best' option would reflect the reasoning of the students and not the conceptual correct explanation to question 4 , since question 5 assess the students' rationale. This was explicitly mentioned to the students for all CRS questions which ask for justifications. Of those students having answered question 4 correctly, only $16 \%$ chose option A for question 5, indicating that this misconception was held by far fewer students than assumed earlier. Another possibility is that option B was more attractive as justification than A, regardless of students' rationale for selecting option A in question 4. Issues with question wording were observed for physics MCQs by Ding et al. ${ }^{[22]}$ For chemistry, interested readers are referred to a series of eight papers ${ }^{[32-39]}$ published over a 26-year span by various authors about a seemingly straight-forward concept question.

Peer instruction was introduced by Mazur. ${ }^{[15,40,41]}$ In short, a question is put to the students, students vote via a CRS, and then discuss their option selection with their neighbors for a few minutes before voting on the same question again. This generally improves the distribution of votes during the subsequent second CRS round of the same question. Further useful information can be 
found from Vickrey et al. ${ }^{[42]}$ and Turpen et al. ${ }^{[43]}$ While it is often a component of the flipped-classroom model, ${ }^{[44]}$ peer instruction can be applied independently. After the first lecture on ICP-MS, which also included a recollection of the ICP, the second lecture started with the following question:

6. In solution iron may occur in different oxidation states. Is it possible to determine the ratio of $\mathrm{Fe}^{2+}$ and $\mathrm{Fe}^{3+}$ in water by ICP-MS? $(N=16)$

$\begin{array}{lll}A & \text { Yes } & 56 \% \\ B^{*} & \text { No } & 37 \% \\ C & \text { Don't know } & 6 \%\end{array}$

Then the students were asked to exchange reasons for their response with their neighbors. Students immediately started to discuss the problem. When it started to get more turbulent, after about 2 minutes, the vote was restarted and the OEQ for reasoning ("Please justify your answer.") was added. For the MCQ the response was $(N=27)$ A (Yes) 56\%, B (No) 33\%, C (Don't know) $11 \%$. Justifications for response A referred to the separation of the mass spectrometer according to the mass-to-charge ratio, a response which was anticipated. Even more surprisingly, the justifications for the correct response B contained only one instance referring to a change of charge stage within the plasma. Most other justifications referred to the small mass of electrons or that both iron species would have the same mass. One student indicated a change of her or his choice for the first question to A. The same sequence of questions was repeated with similar results in following years. Notably, a student who selected option A mentioned ion chromatography within the justification. In this case, option A was the correct choice. To circumvent this ambiguity the stem of the question could be changed to "... by ICP-MS alone?" However, considering that the overwhelming majority of students did not consider this aspect, the additional discriminator is not necessary for a formative format and further subsequent explanation or discussion would be more suitable.

It seems that peer instruction only works if there are enough students in the audience with a sound and subject-specific reasoning and those are able to convince their peers. It is possible that the time for discussion between students was not sufficient to change perspectives, but it is more likely that too few students started with an appropriate reasoning which might even be due to missing information or direct instruction. Occasionally, verbally requested reasoning for choosing a false option resulted only in few responses.

It should be added that there were also instances, whereas peer instruction led to an improved vote distribution for the second run (question 7). However, there are indications that some students may have misread such questions at first (responding to a question that asked for the matrix of chocolate and not the prepared solution) and changed their vote after rereading the question or being made aware of this mistake by their peers. In such cases the first incorrect votes were also not based on misconceptions.

7. What is the matrix of the analyte cadmium in the prepared solution after the digestion of chocolate?

$\begin{array}{lll} & 1 \text { st vote }(N=57) & 2 \text { nd vote }(N=87) \\ A \text { Cacao, sugar, fat } & 65 \% & 33 \% \\ B^{*} \text { Water } & 28 \% & 55 \% \\ C \text { Cadmium ions } & 5 \% & 0 \% \\ D \text { No matrix } & 2 \% & 10 \% \\ E \text { Don't know } & 0 \% & 1 \%\end{array}$

\section{Questions with Justifications}

Given the examples above, three conclusions were drawn: (1) Without further information, assumptions about students' reasoning for selecting an option remained superficial, (2) the fact that students select the correct or best option does not mean they did so for reasons consistent with the accepted scientific model, and (3) the selection of a distractor did not sufficiently indicate a student possesses a misconception. Moreover, a sometimes overlooked issue with diagnosing misconceptions is that mislabeling does not constitute a misconception. ${ }^{[45]}$

On one hand, the CRS question should be followed by an explanation on justifications and possible ambiguities by the lecturer. On the other hand, it is worth to consider asking for students' justifications ${ }^{[46]}$ at least occasionally and for difficult questions. Multiple-tier diagnostic tests have been developed[47] to study misconceptions, which also prompts students' confidence in their response. Furthermore, Chien et al. found evidence that incentives for students to justify their responses is a contributing factor to learning when using CRSs. ${ }^{[48]}$ This also indicates that it is not the CRS itself, which eventually promotes learning, but the questions asked via CRSs. ${ }^{[10]}$ Compared to question-answer sessions with verbal interaction, CRSs offer the advantage to include most if not all students and for the lecturer to likewise receive more representative responses and contemplate on students' justifications. Still, the length of text required for adequate reasoning needs to be taken into account. Prompts for justifications can be incorporated into the CRS questions in different manners, which also assist lecturers in validating students' replies.

Table 1 provides alternatives for prompting justifications with CRS questions. First, note that the question within the stem can be reasonably answered without any options provided (recommendation 3) and it represents a grammatically closed but conceptually open question (ref. [49], see Supporting Information for details). Style 1 is the basic question without any prompt for justification. Style 2 includes justifications in the options, but directly combines them with the responses from 1 . This can be seen either as (a) providing clues or (b) asking mainly for reasoning. The capitalization and underlining is used to put more emphasis on the differences of the options. If justifications ought to be accessed, they should be detached from the basic responses given in style 1. Style 3 provides this by presenting each possible justification for each direct response individually. An alternative is to separate the justification completely from the direct response with a second prompt. This offers the opportunity to introduce a larger variety of possible justifications, since students might not consider certain phrasing or terminology for their reasoning while still employ appropriate models. Still, because style 4 requires already an insight into possible students' reasoning, a prompt for free text input (style 5) can provide a more representative variety. Similarly, but with most common CRSs technically not implemented, the option 'other' in style 4 can be accompanied with the possibility for free text input. Another alternative is to provide a correct statement in the stem and ask only for a justification (style 6). Here, ideally if 'other' is selected students would be able to also respond with additional text, a feature current CRSs do not offer. Tamir found that this format has less diagnostic potential than a well-designed MCQ. ${ }^{[50]}$

In order to contrast the previous styles with an often encountered alternative, one might consider the following: The initial question could be completely rephrased into "Does an incomplete digestion affect the results of repeated analysis randomly? (Yes/No)" (style 7). This avoids the context to the point of the question being 'simple'; it may just require recall, but can also be put effectively to students. The original phrasing is more complex and requires the students to consider the stated settings and conditions. To reiterate the argument above: Whether or not a question is a 'good question' depends on the objectives and other circumstances.

In summary, styles 4 and 5 appear most suited to access students' reasoning directly with CRSs. In contrast to style 4, style 5 requires no insights or well-founded assumption about students' justifications, but the presentation of already phrased options in style 4 make the polling process faster. Style 5 can be routinely 
Table 1. Examples of alternative MCQs to introduce justifications into CRS questions. The correct options are labeled with an asterisk (*), note that justifications in tier 2 may not be labelled as 'correct', because in formative assessment they should reflect the students' rationale.

\section{Item stem \\ A glass shard underwent micro- wave digestion. The result- ing solution is separated into three aliquots and cerium was determined by ICP-OES in each aliquot. The three determined cerium concentrations diverge significantly from each other. Can an incomplete digestion be responsible for that?}

A glass shard underwent microwave digestion. The resulting solution is separated into three aliquots and cerium was determined by ICP-OES in each aliquot. The three determined cerium concentrations diverge significantly from each other. Incomplete digestion CANNOT be responsible for that. Why?

Does an incomplete digestion affect the results of repeated analysis of the solution randomly?

\section{Style}

1. Without justification

2. Justification added to option

3. Multiple justifications for each direct response

\begin{tabular}{l|l} 
4. Separated & $\square$ Yes \\
justification & $\square$ No* \\
as MCQ &
\end{tabular}

5. Separated justifi- $\square$ Yes cation as OEQ $\square$ No* 6. Only justification
(MCQ)

7. Simplified stem and without justification

\section{Question tier 1}

$\square$ Yes

$\square$ YES, because an incomplete digestion affects the cerium concentration in the solutions randomly.

$\square$ NO, because an incomplete digestion affects the cerium concentration in the solutions systematically.*

$\square$ YES, because an incomplete digestion affects the cerium concentration in the solutions systematically.

$\square$ YES, because an incomplete digestion affects the cerium concentration in the solutions randomly.

$\square$ NO, because an incomplete digestion affects the cerium concentration in the solutions systematically.

$\square$ NO, because an incomplete digestion affects the cerium concentration in the solutions randomly.* $\mathrm{No}^{*}$

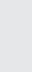

\section{Question tier 2}

Please justify your answer.

$\square$ An incomplete digestion affects the cerium concentration in the solutions randomly.

$\square$ An incomplete digestion affects the cerium concentration in the solutions systematically.

$\square$ Aliquots are taken from the same solution.

$\square$ Other

Please justify your answer.

[free text input required]
$\square$ An incomplete digestion affects the cerium concentration in the solutions randomly.*

$\square$ An incomplete digestion affects the cerium concentration in the solutions systematically.

$\square$ Aliquots are taken from the same solution.*

$\square$ Other

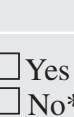

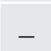


for more effort in this direction in order to bolster active learning in all teaching styles and assist teachers in this regard. To study the suitability of questions in more detail, evaluating justifications is a promising starting point.

\section{Supporting Information}

The supporting information for this article includes elaborations on the structure of questions, their cognitive dimension, best vs. correct options, and specifics to questions in CRSs. URL: https://www.ingentaconnect.com/content/scs/chimia

\section{Acknowledgements}

The author would like to thank Detlef Günther for ample and valuable comments, suggestions, and discussions regarding the manuscript. Sebastian Beck, Urs Brändle, Abigail and Alexander Gundlach Graham, Bodo Hattendorf, Stefanie Ickert, Thomas Korner, Stefan Kradolfer, Christoph Neff, Jens Petersen, and students enrolled in the respective courses, namely Michael Chanton, Vera Ganz, and Monique Kuonen, are gratefully acknowledged for their comments to an early version of the manuscript.

Received: October 26, 2020

[1] Here, the terms question, problem, and task etc. are subsumed under 'question'.

[2] B. S. Bloom, Educational Leadership 1984, 41, 4, https://doi.org/10.2307/1175554

[3] E. Nagy-Shadman, C. Desrochers, Int. J. Sci. Educ. 2008, 30, 2023 , https://www.learntechlib.org/p/71623/.

[4] P. Black, M. Wilson, S. Y. Yao, Measurement-Interdisciplinary Research and Perspectives 2011, 9, 71, https://doi.org/10.1080/15366367.2011.591654

[5] J. Murciano-Calles, J. Chem. Educ. 2020, 97, 4209, https://doi.org/10.1021/acs.jchemed.0c00348

[6] R. J. Pearson, J. Chem. Educ. 2020, 97, 2342, https://doi.org/10.1021/acs.jchemed.0c00464

[7] R. E. Gibbons, E. E. Laga, J. Leon, S. M. Villafane, M. Stains, K. Murphy, J. R. Raker, J. Chem. Educ. 2017, 94, 549, https://doi.org/10.1021/acs.jchemed.6b00799

[8] K. M. Shea, J. Chem. Educ. 2016, 93, 971 https://doi.org/10.1021/acs.jchemed.5b00799

[9] R. W. Morrison, J. A. Caughran, A. L. Sauers, J. Chem. Educ. 2014, 91, 1838, https://www.learntechlib.org/p/153093/

[10] G. Schwarz, 'An Interface Model and Implementation Framework for Classroom Response Systems', submitted to J. Chem. Educ.

[11] T. Tofade, J. Elsner, S. T. Haines, Am. J. Pharmaceut. Educ. 2013, 77, https://doi.org/10.5688/ajpe777155

[12] J. MacArthur, J. Chem. Educ. 2013, 90, 273, https://doi.org/10.1021/ed300215d

[13] R. H. Kay, A. LeSage, Computers \& Education 2009, 53, 819, https://doi.org/10.1016/j.compedu.2009.05.001

[14] S. C. Nurrenbern, W. R. Robinson, J. Chem. Educ. 1998, 75, 1502, https://doi.org/10.1021/ed075p1502

[15] E. Mazur, Science 2009, 323, 50, https://doi.org/10.1126/science.1168927

[16] I. D. Beatty, W. J. Gerace, W. J. Leonard, R. J. Dufresne, Am. J. Phys. 2006, 74, 31, https://doi.org/10.1119/1.2121753

[17] W. J. McKeachie, 'Teaching tips: A guidebook for the beginning teacher', D.C. Heath, 1986.

[18] V. L. Clegg, W. E. Cashin, 'Improving Multiple-Choice Tests, Idea Paper No. 16', Center for Faculty Evaluation and Development, 1986

[19] D. B. King, J. Chem. Educ. 2011, 88, 1485, https://doi.org/10.1021/ ed1004799

[20] E. T. Pappa, G. Tsaparlis, Chem. Educ. Res. Pract. 2011, 12, 262, https://doi.org/10.1039/C1RP90031E

[21] J. Breakall, C. Randles, R. Tasker, Chem. Educ. Res. Pract. 2019, 20, 369 , https://doi.org/10.1039/C8RP00262B

[22] L. Ding, N. W. Reay, A. Lee, L. Bao, Am. J. Phys. 2009, 77, 643, https://doi.org/10.1119/1.3116093

[23] K. D. Schurmeier, C. H. Atwood, C. G. Shepler, G. L. Lautenschlager, J. Chem. Educ. 2010, 87, 1268, https://doi.org/10.1021/ed100422c
[24] J. R. T. Cassels, A. H. Johnstone, J. Chem. Educ. 1984, 61, 613, https://doi.org/10.1021/ed061p613

[25] R. Mitkov, L. E. An Ha, N. Karamanis, Nat. Lang. Engin. 2006, 12, 177

[26] The term 'color' is the hypernym (also umbrella term) of the hyponyms 'red', 'yellow', 'blue', etc.

[27] T. M. Haladyna, S.M. Downing, M.C. Rodriguez, Appl. Meas. Educ. 2002, 15,309 .

[28] M. H. Towns, J. Chem. Educ. 2014, 91, 1426, https://doi.org/10.1021/ed500076x

[29] M. J. Gierl, O. Bulut, Q. Guo, X. Zhang, Rev. Educ. Res. 2017, 87, 1082 , https://doi.org/10.3102/0034654317726529

[30] A. M. Christianson, J. Chem. Educ. 2020, 97, 2701, https://doi.org/10.1021/acs.jchemed.0c00737

[31] National Research Council, 'Science Teaching Reconsidered: A Handbook', 1997, The National Academies Press: Washington, DC., p. 104.

[32] M. J. Sanger, C. K. Vaughn, D. A. Binkley, J. Chem. Educ. 2013, 90, 700, https://doi.org/10.1021/ed084p875

[33] M. S. Cracolice, J.C. Deming, B. Ehlert, J. Chem. Educ. 2008, 85, 873, https://doi.org/10.1021/ed085p873

[34] M. J. Sanger, E. Campbell, J. Felker, C. Spencer, J. Chem. Educ. 2007, 84, 875, https://doi.org/10.1021/ed084p875

[35] M. B. Nakhleh, R. C. Mitchell, J. Chem. Educ. 1993, 70, 190, https://doi.org/10.1021/ed070p190

[36] B. A. Sawrey, J. Chem. Educ. 1990, 67, 253, https://doi.org/10.1021/ed067p253

[37] M. Pickering, J. Chem. Educ. 1990, 67, 254, https://doi.org/10.1021/ed067p254

[38] S. C. Nurrenbern, M. Pickering, J. Chem. Educ. 1987, 64, 508, https://doi.org/10.1021/ed064p508

[39] M. J. Sanger, A. J. Phelps, J. Chem. Educ. 2007, 84, 870, https://doi.org/10.1021/ed084p870

[40] C. H. Crouch, E. Mazur, Am. J. Phys. 2001, 69, 970, https://doi.org/10.1119/1.1374249

[41] K. Miller, N. Lasry, B. Lukoff, J. Schell, E. Mazur, Phys. Rev. Special TopicsPhysics Education Research 2014, 10, 020113 , https://doi.org/10.1103/PhysRevSTPER.10.020113

[42] T. Vickrey, K. Rosploch, R. Rahmanian, M. Pilarz, M. Stains, Cbe-Life Sci. Educ. 2015, 14, es3, https://doi.org/10.1187/cbe.14-11-0198

[43] C. Turpen, N. D. Finkelstein, Phys. Rev. Special Topics - Physics Education Research 2009, 5, 020101. https://doi.org/10.1103/PhysRevSTPER.5.020101

[44] F. Ponikwer, B. A. Patel, Anal. Bioanal. Chem. 2018, 410, 2263, https://doi.org/10.1007/s00216-018-0892-2

[45] D. Clerk, M. Rutherford, Int. J. Sci. Educ. 2000, 22, 703, https://doi.org/10.1080/09500690050044053

[46] P. Tamir, Int. J. Sci. Educ. 1990, 12, 563, https://doi.org/10.1080/0950069900120508

[47] D. D. Milenković, T. N. Hrin, M. D. Segedinac, S. Horvat, J. Chem. Educ 2016, 93, 1514, https://doi.org/10.1021/acs.jchemed.6b00261

[48] Y. T. Chien, Y. H. Chang, C. Y. Chang, Educ. Res. Rev. 2016, 17, 1, https://doi.org/10.1016/j.edurev.2015.10.003

[49] P. Worley, J. Philos. Schools 2015, 2, 17, http://doi.org/10.21913/jps. $\mathrm{v} 2 \mathrm{i} 2.1269$

[50] P. Tamir, J. Biol. Educ. 1989, 23, 285, https://doi.org/10.1080/00219266.1989.9655083

[51] J. P. A. Prieto, A. H. Escobar, 'Exit Tickets' Effect on Engagement in College Classrooms', Edulearn16: 8th International Conference on Education and New Learning Technologies, 2016, pp 5915-5918.

[52] A. Sprague, Tesol J. 2016, 7, 989, https://doi.org/10.1002/tesj.295

[53] S. R. Leigh, Int. J. Teach. Learn. Higher Educ. 2012, 24, 189, http://www.isetl.org/ijtlhe/

[54] R. J. Marzano, Educational Leadership 2012, 70, 80.

\section{License and Terms}

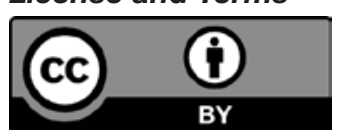

This is an Open Access article under the terms of the Creative Commons Attribution License CC BY 4.0. The material may not be used for commercial purposes.

The license is subject to the CHIMIA terms and conditions: (http:// chimia.ch/component/sppagebuilder/?view=page \&id=12).

The definitive version of this article is the electronic one that can be found at https://doi.org/10.2533/chimia.2021.33 